\title{
Post-Operative Malignant Hyperthermia Complicated by Rhabdomyolysis: Case Report ${ }^{*}$
}

\author{
Kenji Yamada ${ }^{1}$, Tadao Okada ${ }^{2 \#}$, Shohei Honda ${ }^{2}$, Hisayuki Miyagi ${ }^{2}$, Hitoshi Ono ${ }^{1}$, Yuji Morimoto ${ }^{3}$, \\ Hiroaki Yaguchi ${ }^{4}$, Toshi Tugawa ${ }^{5}$, Akinobu Taketomi ${ }^{1}$ \\ ${ }^{1}$ Department of Gastroenterological Surgery I, Graduate School of Medicine, Hokkaido University, Sapporo, Japan \\ ${ }^{2}$ Department of Pediatric Surgery, Hokkaido University Hospital, Sapporo, Japan \\ ${ }^{3}$ Department of Anesthesia, Graduate School of Medicine, Hokkaido University, Sapporo, Japan \\ ${ }^{4}$ Department of Neurology, Graduate School of Medicine, Hokkaido University, Sapporo, Japan \\ ${ }^{5}$ Department of Pediatrics, Saiseikai Nishi Otaru Hospital, Otaru, Japan \\ Email:"okadata@med.hokudai.ac.jp
}

Received October 7, 2011; revised December 4, 2011; accepted December 19, 2011

\begin{abstract}
Malignant hyperthermia (MH) is a rare but fatal complication that develops under general anesthesia. Particularly, reports of postoperative $\mathrm{MH}$ are rare. This report describes the very rare case of a 29 -year-old woman with neurological impairment complicated by rhabdomyolysis due to postoperative MH with an onset 30 hours after laparoscopic Nissen fundoplication and open gastrostomy, and the successful medical treatment of $\mathrm{MH}$. We review the literature on this type of relationship between postoperative $\mathrm{MH}$ and neurological impairment, and discuss the clinical features of this complication. Furthermore, the usefulness of magnetic resonance imaging for the diagnosis of rhabdmyolysis caused by postoperative MH was explored.
\end{abstract}

Keywords: Postoperative Malignant Hyperthermia; Neurological Impairment; MRI; Nissen Fundoplication

\section{Introduction}

The prevalence of malignant hyperthermia (MH) is reportedly 1:50,000 - 250,000 during general anesthesia. With the introduction of dantrolene, mortality due to $\mathrm{MH}$ has decreased significantly [1], but $\mathrm{MH}$ is still a life-threatening condition, with a mortality of $15 \%$ in patients with fulminant MH (f-MH). The onset of MH is usually during general anesthesia. Postoperative MH is defined as that after general anesthesia. The prevalence of postoperative $\mathrm{MH}$ is reportedly $1.9 \%$ of $\mathrm{MH}$ and begins shortly after the complication of anesthetic care [2].

We herein report a case of rhabdomyolysis caused by postoperative $\mathrm{MH}$ associated with an onset 30 hours after laparoscopic Nissen fundoplication and open gastrostomy for a 29-year-old woman with neurological impairment. We review the literature on this type of disorder, and discuss the problems associated with the characteristics of children with rhabdmyolysis due to postoperative MH with neurological impairment, particularly concerning the importance of MRI in the diagnosis of rhabdomyolysis.

\footnotetext{
"This study was not supported by any grants. Source of Support: Nill. Conflict of Interest: None decleared.

"Corresponding author.
}

\section{Case Report}

A 29-year-old woman, weighing $16.9 \mathrm{~kg}$ and $113 \mathrm{~cm}$ tall, was admitted to our hospital due to eating disorder and gastroesophageal reflux. The patient had scoliosis. Her medical history showed marked mental retardation, cerebral palsy, and contracture due to neuroaxal dystrophy. There was neither a personal nor family history of muscular dystrophy or MH. She had no history of drug allergies. The results of preoperative laboratory tests showed: aspartate aminotransferase, $31 \mathrm{U} / \mathrm{L}$; alanine aminotransferase, $26 \mathrm{U} / \mathrm{L}$; blood urea nitrogen, $17 \mathrm{mg} / \mathrm{dl}$; serum creatinine, $0.4 \mathrm{mg} / \mathrm{dl}$; and creatine kinase, $528 \mathrm{IU} / \mathrm{L}$ (normal: 45 - $163 \mathrm{IU} / \mathrm{L}$ ).

The patient was taken to the operation room for laparoscopic Nissen fundoplication and open gastrostomy, and a rapid sequence induction for general anesthesia was performed. The patient received rocronium at $15 \mathrm{mg}$ and propofol at $200 \mathrm{mg}$ for general induction. She was maintained with oxygen and sevoflurane anesthesia in addition to remifentanyl. The body temperature during general anesthesia ranged from $36.0^{\circ} \mathrm{C}$ to $36.5^{\circ} \mathrm{C}$. The operation time was 3 hours and 47 minutes, and the period of general anesthesia was 5 hours and 33 minutes. The patient was exubated and returned to her bed with no problems.

On the night of the next day after the operation at postoperative 30 hours, the patient developed hyperthermia 
of $39.0^{\circ} \mathrm{C}$ accompanied by tachycardia $(130 \mathrm{bpm} / \mathrm{min})$ which was resistant to acetaminophen. We suspected respiratory infection because she showed Pseudomonas aeruginosa on the preoperative culture of a pharyngeal specimen. Therefore, the patient received intravenous antibiotic therapy.

On the second day after the operation at postoperative 40 hours, the patient developed hyperthermia of $41.1^{\circ} \mathrm{C}$ followed by anuria and hematuria (Figure 1). The initial work-up revealed a markedly elevated creatinine kinase level of $7770 \mathrm{IU} / \mathrm{L}$, abnormal urine myoglobin level of $37,000 \mathrm{ng} / \mathrm{ml}$ (normal $0-10 \mathrm{ng} / \mathrm{ml}$ ), and serum myogloin level of $9057.3 \mathrm{mg} / \mathrm{ml}$ (normal $0-60 \mathrm{ng} / \mathrm{ml}$ ). The serum calcium level was $9.8 \mathrm{mg} / \mathrm{dl}$, the blood urine nitrogen level was $49 \mathrm{mg} / \mathrm{dl}$, and creatinine was $0.9 \mathrm{mg} / \mathrm{dl}$. Plain computed tomography showed no chest effusion, pneumonia, or abdominal mass. We diagnosed her with rhabdomyolysis and renal failure caused by postoperative MH based on Morio's criteria [3]. To manage general deterioration due to postoperative $\mathrm{MH}$, the patient was transferred to the intensive care unit (ICU). She received hymdration of $20 \mathrm{ml} / \mathrm{kg} / \mathrm{h}$ of Ringer's lactate and repeated intravenous dantrolene $(0.5 \mathrm{mg} / \mathrm{kg})$ for 6 hours for the treatment of MH. Two hours after being transferred to the ICU, urine output increased, and the patient became afebrile at 15 hours after transfer to the ICU.

Magnetic resonance imaging (MRI) using a short-time inversion recovery sequence (STIR) was performed on the whole body for the diagnosis of rhabdomyolysis. MRI demonstrated a high intensity in the deltoid muscles (Figure 2(a)). These findings were consistent with rhabdomyolysis. On the fifth day after the operation, the patient returned to the ward. She showed no high fever, and urine

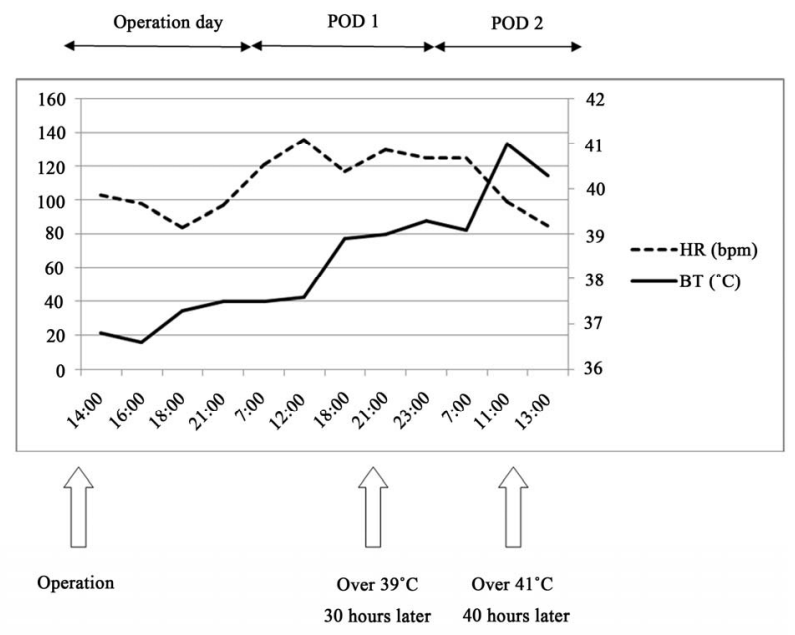

POD: postoperation day $\quad$ HR: heart rate $\quad$ BT: body temperature

Figure 1. Heart rate and body temperature over the postoperative course. At $\mathbf{3 0}$ hours postoperatively, the patient developed hyperthermia of $39.0^{\circ} \mathrm{C}$ followed by tachycardia (130 bpm/min), and, at 40 hours postoperatively, she developed hyperthermia of $41.1^{\circ} \mathrm{C}$. and serum myoglobin improved to 30.3 and $272.6 \mathrm{ng} / \mathrm{dl}$, respectively (Table 1).

The patient was discharged at 46 days after the operation. MRI of the deltoid muscles performed on the day of discharge was normal (Figure 2(b)).

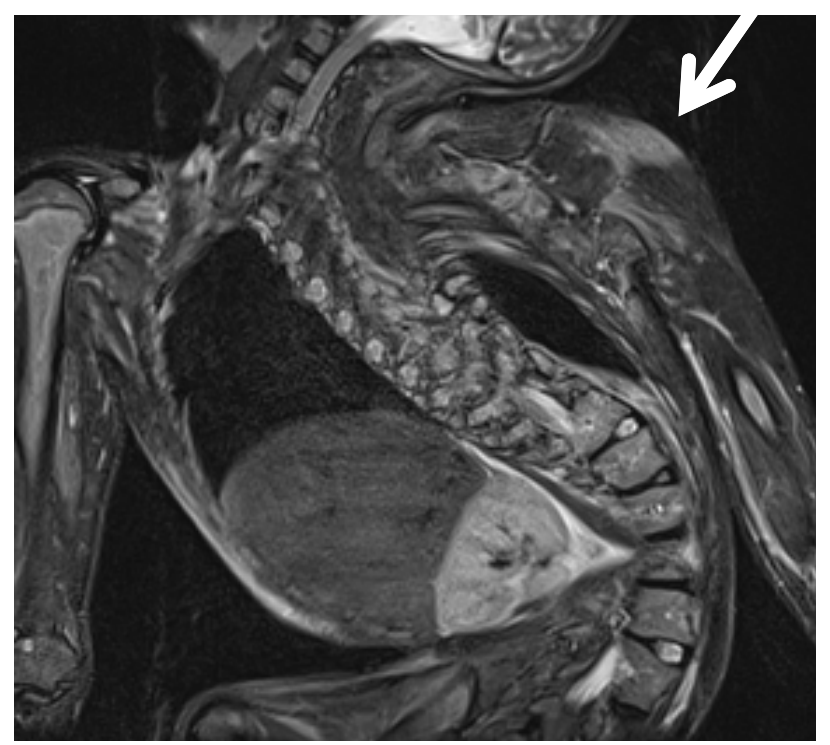

(a)

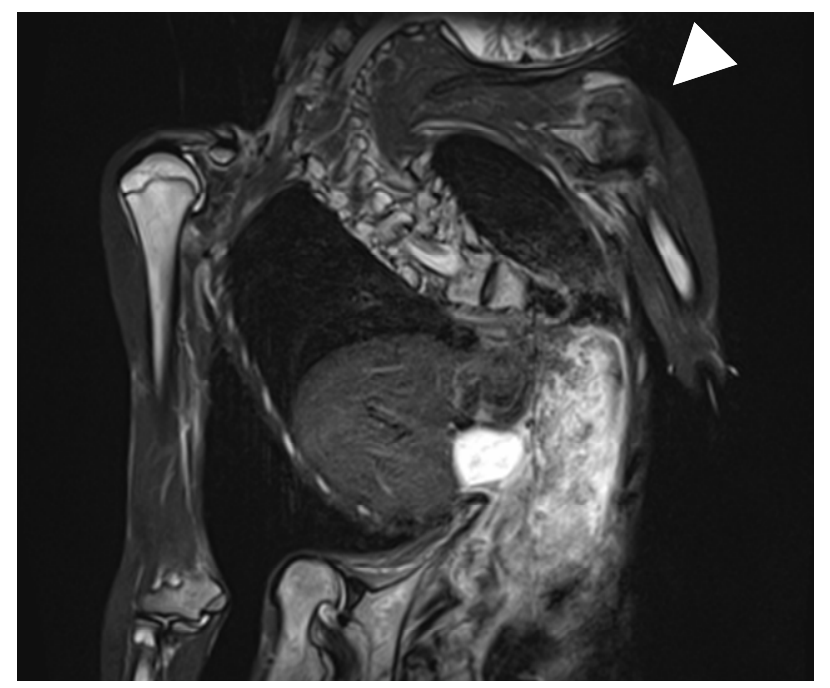

(b)

Figure 2. MRI using STIR, (a) The finding indicating rhabdomyolysis caused by postoperative MH was a high intensity (arrow)in the deltoid muscles; (b) MRI using STIR obtained from the same deltoid muscles on the day of discharge was normal (arrowhead).

Table 1. Serum and urine myoglobin levels over the postorative course.

\begin{tabular}{ccccc}
\hline & POD 2 & POD 5 & POD 12 & POD 26 \\
\hline Serum myoglobin $(\mathrm{ng} / \mathrm{ml})$ & 9057.3 & 1934.6 & 359.7 & 272.6 \\
urine myoglobin $(\mathrm{ng} / \mathrm{ml})$ & 37,000 & 4436.3 & 39.1 & 30.3 \\
\hline
\end{tabular}




\section{Discussion}

$\mathrm{MH}$ is a pharmacogenetic disorder of skeletal muscle that presents as a hypermetabolic response to potent volatile anesthetic gases such as halothane, sevoflurane, and the depolarizing muscle relaxant succinylcholine, and rarely, in humans, to stress such as vigorous exercise and heat [4]. Early MH signs include masseter muscle rigidity, a rise in endtidal $\mathrm{CO}_{2}$, acidosis, and unexpected tachycardia due to hypermetabolism [5]. Succeeding signs include an increased body temperature, which is the origin of the term "MH", and ventricular arrhythmia. As late signs of $\mathrm{MH}$, continuous muscle contractions may provoke generalized muscle rigidity, which is typically followed by rhabdomyolysis when inappropriately treated [5]. Late signs also include cardiac arrest with hyperpotassemia, colacolored urine, and a rise in creatine kinase (CK) [5].

We used the diagnostic criteria of Morio et al. [3] for the diagnosis of $\mathrm{MH}$ in our patient, who was classified as f-MH due to significant hyperthermia over $41^{\circ} \mathrm{C}$. The clinical grading scale (CGS) of Larach et al. [6] for MH is divided into 1 - 6 categories. According to the CGS, our patient's clinical characteristic was category 5 "very likely", and MH was strongly suspected. For a definitive diagnosis, muscle biopsy is necessary to detect a genetic mutation of the ryanodine receptor, RYR1, a calcium channel receptor which controls the release of calcium from sarcoplasmic reticulum [7]. However, we could not obtain consent for muscle biopsy from her family. Therefore, the diagnosis of $\mathrm{MH}$ was made based on the clinical diagnostic criteria and CGS.

Although the onset of $\mathrm{MH}$ is rapid after the induction of anesthesia in most reported cases with $\mathrm{MH}$, it took 30 hours after the operation in the present case. We initially suspected respiratory infection at 30 hours postoperation, followed by a delay in the diagnosis of MH. The early diagnosis and initial treatment for $\mathrm{MH}$ are important prognostic factors; the mortality rate due to $\mathrm{MH}$ is $2.6 \%$ when the maximum body temperature is less than $40^{\circ} \mathrm{C}$, but the mortality rises to $53 \%$ when the maximum temperature exceeds $41^{\circ} \mathrm{C}$, such as in the present case [8]. Therefore, a prompt reduction in the body temperature from hyperthermia is important to improve the prognosis.

In the present case, we thought that the reasons for the delayed onset of MH involved the small amount of skeletal muscle and stress of pain associated with the operation. It is assumed that MH tends to occur in people that have a large volume of skeletal muscle and a higher metabolic rate, and the symptoms become apparent at several hours after induction [7]. The amount of skeletal muscle in the present case was lower than normal because she was $\leq-2$ SD of the average weight. Therefore, it remains possible that the onset of $\mathrm{MH}$ was delayed in our patient. Another theory for the late onset of MH is the involvement of the stress of pain associated with the opera- tion in patients with neurological impairment. Sayo et al. [9] started that pain control was an important intervention to decrease the postoperative stress leading to $\mathrm{MH}$ for children with neurological impairment in the perioperative period. Stress in the postoperative period might have been a factor triggering $\mathrm{MH}$ in the present case. Based on this theory, it is important to closely follow patients with neurological impairment for the early detection of several signs of $\mathrm{MH}$, even in the postoperative period.

MRI was useful for the diagnosis of rhabdomyolysis in the present case. Moratalla MB et al. [10] referred to the importance of MRI in the diagnosis of rhabdomyolysis. STIR images of MRI display a good contrast between normal and abnormal muscles and better differentiation of the damaged muscles from the adjacent fat due to its fat suppression [10]. In the present case, a high signal intensity was present on STIR due to rhabdomyolysis caused by postoperative MH. The sensitivity of MRI of the extremities to detect abnormal muscles is higher than that of CT or ultrasound ( $100 \%, 62 \%$, and $42 \%$, respectively) in patients with rhabdomyolysis [11]. Furthermore, for signal abnormalities on MRI might persist for longer compared with the improvement of clinical signs of $\mathrm{MH}$ [11].

\section{Conclusions}

We experienced an episode of postoperative $\mathrm{MH}$ in a patient with neurological impairment. In conclusion, our experience suggests that MRI of the extremities provides an accurate tool for the diagnosis of rhabdmyolysis in patients with postoperative MH. Furthermore, we need to consider that $\mathrm{MH}$ can occur after surgery even in patients even with neurological impairment, especially with unusual postoperative hyperthermia.

\section{REFERENCES}

[1] K. P. Strazis and A. W. Fox, "Malignant Hyperthermia: A Review of Published Cases," Anesthesia \& Analgesia, Vol. 77, No. 2, 1993, pp. 297-304. doi:10.1213/00000539-199308000-00014

[2] R. S. Litman, C. D. Flood, R. F. Kaplan, Y. L. Kim and J. R. Tobin, "Postoperative Malignant Hyperthermia: An Analysis of Cases from the North American Malignant Hyperthermia Registry," Anesthesiology, Vol. 109, No. 5, 2008, pp. 825-829. doi:10.1097/ALN.0b013e31818958e5

[3] M. Morio, H. Kikuchi and O. Yuge, "Clinical Criteria of Malignanthyperthermia. Revisedcriteria (in Japanese)," Masui to Sosei, Vol. 19, 1998, pp. 104-110.

[4] R. S. Litman and H. Rosenberg, "Malignant Hyperthermia: Update on Susceptibility Testing," Journal of the American Medical Association, Vol. 293, No. 23, 2005, pp. 2918-2924. doi:10.1001/jama.293.23.2918

[5] T. Migita, K. Mukaida, M. Kawamoto, M. Kobayashi and 
O. Yuge, "Fulminant-Type Malignant Hyperthermia in Japan: Cumulative Analysis of 383 Cases," Journal of Anesthesia, Vol. 21, No. 2, 2007, pp. 285-288. doi:10.1007/s00540-006-0495-5

[6] M. G. Larach, A. R. Localio, G. C. Allen, M. A. Denborough, F. R. Ellis, G. A. Gronert, R. F. Kaplan, S. M. Muldoon, T. E. Nelson and H. Ording, "A Clinical Grading Scale to Predict Malignant Hyperthermia Susceptibility," Anestesiology, Vol. 80, No. 4, 1994, pp. 771-779. doi:10.1097/00000542-199404000-00008

[7] N. Nakamura, T. Ueda, R. Ishikawa, Y. Tasaka, K. Fukuuchi and N. Sato, "Malignant Hyperthermia Developing during Esophageal Resection in an 82-Year-Old Man," Journal of Anesthesia, Vol. 22, No. 4, 2008, pp. 464-466. doi:10.1007/s00540-008-0641-3

[8] Y. Maehara, K. Mukaida, M. Kawamoto and O. Yuge, "An Analysis of Fatal Malignant Cases after 1990 in Ja- pan (in Japanese with English Abstract)," Japan Society for Clinical Anesthesia, Vol. 20, 2000, pp. 385-390. doi:10.2199/jijsca.20.385

[9] S. Nagai, J. Nakata, K. Funaki, T. Harada and R. Watanabe, "Postoperative Pain Management for Nissen Fundoplication in Severely Retaeded Children: 5 Cases Reports (in Japanese)" Clinical Pediatric Anesthesia, Vol. 12, No. 1, 2006, pp. 114-117.

[10] M. B. Moratalla, P. Braun and G. M. Fornas, "Importance of MRI in the Diagnosis and Treatment of Rhabdomyolysis," European Journal of Radiology, Vol. 65, No. 2, 2008, pp. 311-315. doi:10.1016/j.ejrad.2007.03.033

[11] C. S. Restrepo, D. F. Lemos, H. Gordillo, R. Odero, T. Varghese, W. Tiemann, F. F. Rivas, R. Moncada and C. R. Gimenez, "Imaging Findings in Musculoskeletal Complication of AIDS," Radiographics, Vol. 24, No. 4, 2004, pp. 1029-1049. doi:10.1148/rg.244035151 